Return to the Manage Active Submissions page at http://spie.org/app/submissions/tasks.aspx and approve or disapprove this submission. Your manuscript will not be published without this approval. Please contact author_help@spie.org with any questions or concerns.

\title{
Comparative study of Cr:Nd:YAG and Nd:YAG solar laser performances
}

\author{
D. Liang*, J. Almeida and D. Garcia \\ CEFITEC, Departamento de Física, FCT, Universidade Nova de Lisboa, 2829-516, \\ Campus de Caparica, Portugal \\ *d1@fct.unl.pt, jla@campus.fct.unl.pt,dm.garcia@campus.fct.unl.pt
}

\begin{abstract}
To improve the efficiency of $\mathrm{Nd}^{3+}$-doped YAG solar laser, cross-pumped $\mathrm{Cr}^{3+}$ and $\mathrm{Nd}^{3+}$ co-doped YAG ceramic material has attracted more attentions in recent years. The sensitizer $\mathrm{Cr}^{3+}$ ions have broad absorption bands in the visible region. Despite the interests in Cr:Nd:YAG ceramic medium, researchers have achieved significant laser efficiencies with different Nd:YAG single-crystal rods. While it is clear about the effectiveness of Nd:YAG single-crystal rods for solar laser operation, there still exist some concerns about the advantages of Cr:Nd:YAG ceramics in solar-pumped lasers. A $0.9 \mathrm{~m}$ diameter Fresnel lens is used as an economical solar collector. A $4 \mathrm{~mm}$ diameter, $25 \mathrm{~mm}$ length 1.0 at $\%$ $\mathrm{Nd}: Y A G$ single-crystal rod and a 0.1 at $\% \mathrm{Cr}: 1.0$ at $\% \mathrm{Nd}: Y A G$ ceramic rod are pumped alternatively within a conical cavity through a secondary concentrator. With the Nd:YAG rod, the maximum laser power is $12.3 \mathrm{~W}$, corresponding to $19.3 \mathrm{~W} / \mathrm{m}^{2}$ collection efficiency. With the Cr:Nd:YAG ceramic rod, the maximum laser power is $13.5 \mathrm{~W}$, corresponding to $21.2 \mathrm{~W} / \mathrm{m}^{2}$ collection efficiency. This result is, to the best of our knowledge, the highest collection efficiency achieved with Cr:Nd:YAG ceramic medium. There is also a 109\% increase in slope efficiency. In summary, we have experimentally observed a moderate, but not significant, advantage of $\mathrm{Cr}$ :Nd:YAG ceramics over Nd:YAG single-crystal medium in both solar laser conversion and slope efficiency.
\end{abstract}

Keywords: solar laser, Fresnel lens, Nd:YAG, Cr:Nd:YAG, collection efficiency, brightness

\section{INTRODUCTION}

Solar-pumped lasers have gained an ever-increasing importance in recent years [1]. Compared to electrically powered lasers, solar laser is much simpler and more reliable due to the complete elimination of the electrical power generation and conditioning equipments. This technology has a large potential for many applications, e.g. high-temperature materials processing, free space laser communications, space to earth power transmission, and so on. The renewable recovery of $\mathrm{Mg}$ from $\mathrm{MgO}$ is another very interesting topic for solar-pumped lasers. Ultra-high brightness renewable solar-pumped laser beams can be very conveniently focused to heat the magnesium oxide to more than $4000 \mathrm{~K}$ and thus create pure magnesium. Magnesium can be easily stored and transported in the form of "pellets" and, when necessary, reacts with water to produce both hydrogen and thermal energy for fuel cell vehicles and other applications.

The first solar-pumped laser was reported by Young in 1966 [2]. Since then, researchers have been exploiting both parabolic mirrors and Fresnel lenses to attain enough concentrated solar radiation at focal point and several pumping schemes have been constructed for enhancing solar laser output performances [1-14]. To improve the efficiency of $\mathrm{Nd}^{3+}-$ doped YAG laser, cross-pumped $\mathrm{Cr}^{3+}$ and $\mathrm{Nd}^{3+}$ co-doped YAG ceramic material has attracted more attentions in recent years. The sensitizer $\mathrm{Cr}^{3+}$ ions have broad absorption bands in the visible region. By the ${ }^{4} \mathrm{~T}_{2}$ to ${ }^{4} \mathrm{~A}_{2}$ transition of $\mathrm{Cr}^{3+}$ ions, energy is transferred from $\mathrm{Cr}^{3+}$ to $\mathrm{Nd}^{3+}$ ions. For single-shot laser operation with a $0.1 \mathrm{at}^{\%} \mathrm{Cr}^{3+}$ and $1.0 \mathrm{at} \% \mathrm{Nd}^{3+}$ codoped YAG ceramic rod, the laser efficiency is found to be more than twice that of a $1.0 \mathrm{at} \% \mathrm{Nd}^{3+}$ :YAG ceramic rod. At low repetition rates, the average output power of Cr:Nd:YAG rod is higher than that of Nd:YAG. However, this tendency gradually decreases with increasing repetition rates [15]. Despite the interests in Cr:Nd:YAG ceramic medium, researchers have achieved significant laser efficiencies with different Nd:YAG single-crystal rods [10-12]. 19.3 W/m² collection efficiency has been reported by us [10] with a $4 \mathrm{~mm}$ diameter, $25 \mathrm{~mm}$ length Nd:YAG single-crystal rod 
Return to the Manage Active Submissions page at http://spie.org/app/submissions/tasks.aspx and approve or disapprove this submission. Your manuscript will not be published without this approval. Please contact author_help@spie.org with any questions or concerns.

pumped through a $0.9 \mathrm{~m}$ diameter Fresnel lens. The most recent solar-pumped laser with a liquid light guide lens and also a $6 \mathrm{~mm}$ diameter, $100 \mathrm{~mm}$ length $\mathrm{Nd}: \mathrm{YAG}$ rod has produced $30.0 \mathrm{~W} / \mathrm{m}^{2}$ collection efficiency [12], despite its very low laser beam brightness figure of merit $\mathrm{B}=6.6 \times 10^{-3} \mathrm{~W}$. Due to the strong thermal load, the collection efficiency with the Nd:YAG rod is unexpectedly much better than that with Cr:Nd:YAG ceramic rod [12]. While it is clear about the effectiveness of Nd:YAG single-crystal rods for solar laser operation, there still exist, in our opinion, some concerns about the advantages of $\mathrm{Cr}: \mathrm{Nd}: Y A G$ ceramic medium in solar-pumped lasers.

To clarify the advantages of Cr:Nd:YAG ceramics rods in solar-pumped lasers, a fused silica light guide with rectangular cross-section is coupled to a compound V-shaped cavity within which a $7 \mathrm{~mm}$ diameter, $30 \mathrm{~mm}$ length, 0.1 at $\%$ Cr: 1.0 at $\%$ Nd:YAG ceramic rod is uniformly pumped [13]. The highly concentrated solar radiation at the focal spot of a $2 \mathrm{~m}$ diameter stationary parabolic mirror is transformed into a uniform pump radiation by the light guide. Efficient pump light absorption is achieved by pumping uniformly the ceramic rod within the $\mathrm{V}$-shaped cavity. Optimum pumping parameters and solar laser output powers are found through $\mathrm{ZEMAX}^{\odot}$ non-sequential ray-tracing and $\mathrm{LASCAD}^{\odot}$ laser cavity analysis codes. $33.6 \mathrm{~W}$ continuous-wave laser power is measured, corresponding to 1.32 times enhancement over our previous results with a $4 \mathrm{~mm}$ diameter, $30 \mathrm{~mm}$ length Nd:YAG single-crystal rod. High slope efficiency of $2.6 \%$ is also registered. The solar laser output performances of both the ceramic and the single-crystal rods are finally compared, revealing the relative advantage of the Cr:Nd:YAG rod in conversion efficiency. Low scattering coefficient of 0.0018 $\mathrm{cm}^{-1}$ is deduced for the ceramic rod. Heat load is considered as a key factor affecting the ceramic laser output performance.

To compare the solar laser output performances of both Cr:Nd:YAG and Nd:YAG rods in end-pumping configuration, a $0.9 \mathrm{~m}$ diameter Fresnel lens is used as an economical solar collector, as shown in Fig. 1 . The $4 \mathrm{~mm}$ diameter, $25 \mathrm{~mm}$ length, $1.0 \mathrm{at} \% \mathrm{Nd}: Y A G$ single-crystal rod and the $0.1 \mathrm{at} \% \mathrm{Cr}: 1.0$ at $\% \mathrm{Nd}: \mathrm{YAG}$ ceramic rod are pumped alternatively within the conical cavity through a secondary concentrator, as shown in Fig.2. With the Nd:YAG rod, the maximum laser power is $12.3 \mathrm{~W}$, corresponding to $19.3 \mathrm{~W} / \mathrm{m}^{2}$ collection efficiency. With the Cr:Nd:YAG ceramic rod, the maximum laser power is $13.5 \mathrm{~W}$, corresponding to $21.2 \mathrm{~W} / \mathrm{m}^{2}$ collection efficiency. This result is, to the best of our knowledge, the highest collection efficiency achieved with Cr:Nd:YAG ceramic medium. There is also a $109 \%$ increase in slope efficiency by using the ceramic rod.

\section{HIGH COLLECTION EFFICIENCY Nd:YAG AND Cr:Nd:YAG SOLAR LASER SYSTEM}

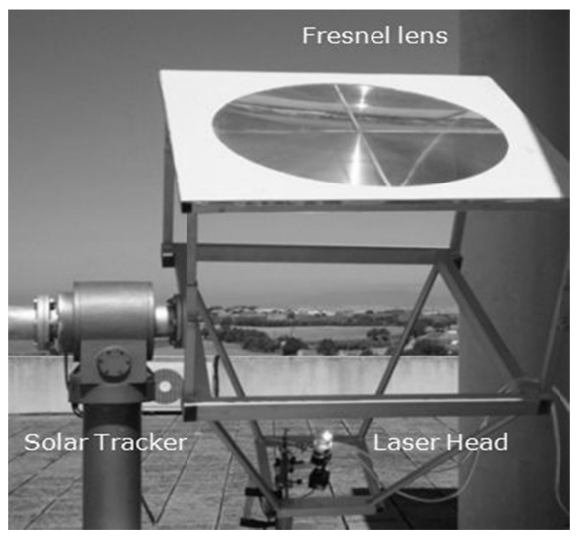

Fig.1. The Nd:YAG, Cr:Nd:YAG solar-pumped laser system with a 0.9 m diameter Fresnel lens and a laser head positioned in its focal zone.

As shown in Fig.1, the Nd:YAG solar-pumped laser system is composed of the $0.9 \mathrm{~m}$ diameter Fresnel lens mounted on a two-axis solar tracker that follows automatically the sun's movement. The solar tracker is supplied by Shandong Huayi Sunlight Solar Energy Industry Co., Ltd. The Fresnel lens (NTK CF1200) is supplied by Nihon Tokushu Kogaku Jushi Co., Ltd. The Fresnel lens is made of Polymethyl Methacrylate (PMMA) material, which is transparent at visible and 
Return to the Manage Active Submissions page at http://spie.org/app/submissions/tasks.aspx and approve or disapprove this submission. Your manuscript will not be published without this approval. Please contact author help@spie.org with any questions or concerns.

near infrared wavelengths, but absorbs the infrared radiation beyond $2200 \mathrm{~nm}$ and cut undesirable UV solar radiation below $350 \mathrm{~nm}$. We find that $78.6 \%$ of incoming solar radiation is focused to the focal zone about $1.2 \mathrm{~m}$ away from the Fresnel lens. In fact, the concentrated solar power at the focal point averaged over $2 \mathrm{~min}$ is $445 \mathrm{~W}$ for source sunlight of $890 \mathrm{~W} / \mathrm{m}^{2}$. The measured full width at half maximum beam waist is about $12 \mathrm{~mm}$. Mechanical adjustments in X-Y-Z directions allow the alignment of the laser head in the focal zone. Coarse angular adjustments around $\mathrm{X}, \mathrm{Y}$ and $\mathrm{Z}$ axis are also helpful for achieving the maximum laser output power. Fresnel lens is cost-effective, but the largely dispersed radiation distributed along its focal zone hampers further efficient light concentration to a thin rod. For this reason, a novel secondary laser pumping scheme becomes indispensable. Dielectric totally internally reflecting concentrator (DTIRC) was originally designed for photovoltaic applications $[16,17]$. A modified version of the DTIRC with a large curved input face and a small planar output face is utilized by us to concentrate efficiently the solar radiations from the focal zone to the thin rod, which is mounted within the conical pumping cavity. Cooling water also plays a crucial role in ensuring efficient light coupling between the output end of the DTIRC and the rod. Efficient absorption of pump radiation is achieved through both direct end-pumping and multi-pass side-pumping within the cavity. Optimized parameters of the Fresnel lens, the DTIRC and the conical cavity are found by non-sequential ray-tracing code $\left(\right.$ ZEMAX $\left.^{\mathrm{TM}}\right)$. Optimized laser resonator parameters are obtained by laser cavity design and analysis code (LASCAD $\left.{ }^{\mathrm{TM}}\right)$.

The laser head is composed of the DTIRC coupled to the conical pumping cavity where either the $4 \mathrm{~mm}$ diameter, $25 \mathrm{~mm}$ length, Nd:YAG single-crystal rod or the $4 \mathrm{~mm}$ diameter. $25 \mathrm{~mm}$ length, Cr:Nd:YAG ceramic rod is mounted. Chromatic aberration of the Fresnel lens spread the focal spots of different wavelengths along its large focal region, so it is difficult to couple efficiently all the concentrated radiations within this region into a small laser rod by a conventional lens. The modified DTIRC is therefore designed to overcome this difficulty. The large curved input face is designed to both collect and compress the concentrated solar radiation from the large focal zone to the upper end face of the laser, as indicated in Fig.2. For end-pumping, one part of the radiation, represented by ray A, is focused onto the upper end face of the rod by either the direct focusing from the curved face or also by the indirect total internal reflections from the side walls of the DTIRC, as represented by ray B.

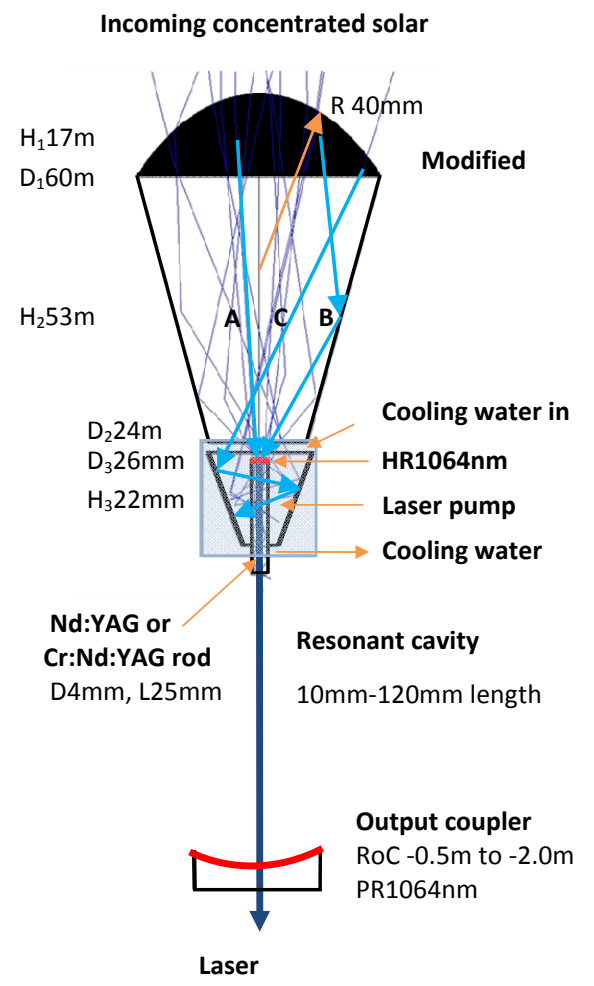

Fig.2. The Nd:YAG, Cr:Nd:YAG laser head is composed of the DTIRC coupled to the conical pump cavity where either the $4 \mathrm{~mm}$ diameter Nd:YAG single-crystal rod or Cr:Nd:YAG ceramic rod is efficiently pumped. A concave output coupler is optically aligned along the rod axis. 
Return to the Manage Active Submissions page at http://spie.org/app/submissions/tasks.aspx and approve or disapprove this submission. Your manuscript will not be published without this approval. Please contact author help@spie.org with any questions or concerns.

For side-pumping, another part of the radiation not hitting the end face of the rod, represented by ray $\mathrm{C}$, is also guided into the small conical cavity with $\mathrm{D}_{3}=26 \mathrm{~mm}$ input diameter, $\mathrm{D}_{4}=10 \mathrm{~mm}$ output diameter and $\mathrm{H}_{3}=22 \mathrm{~mm}$ cavity height. The zigzag passage of the rays within the cavity ensures a multi-pass side-pumping to the rod. All the design parameters in Fig.2 are optimized by ZEMAX ${ }^{\mathrm{TM}}$ non-sequential ray-tracing software in order to obtain the maximum absorbed pump power within the Nd:YAG rod. To enhance light coupling efficiency, the laser head is mounted $1.17 \mathrm{~m}$ away from the Fresnel lens, $3 \mathrm{~cm}$ above the beam waist position of the focal zone. To maximize the output laser power, laser resonator parameters with $-0.5 \mathrm{~m}$ radius of curvature $(\mathrm{RoC})$ and $120 \mathrm{~mm}$ cavity length are obtained by LASCAD ${ }^{\mathrm{TM}}$ laser cavity design and analysis code. As shown in Fig.2, the laser cavity length can be varied from $10 \mathrm{~mm}$ to $120 \mathrm{~mm}$. It can also be extended to more than $200 \mathrm{~mm}$. The $\mathrm{M}^{2}$ factor can be largely reduced in this case.

To manufacture the DTIRC, a $99.995 \%$ optical purity fused silica rod with $60 \mathrm{~mm}$ diameter and $100 \mathrm{~mm}$ length is supplied by Beijing Kinglass Quartz Co., Ltd. It is firstly ground and polished to form the curved input face with $\mathrm{R}=40$ $\mathrm{mm}, \mathrm{D}_{1}=60 \mathrm{~mm}$ diameter and $\mathrm{H}_{1}=17 \mathrm{~mm}$ height. The conical section of the DTIRC is then machined and polished to the dimensions of $\mathrm{D}_{1}=60 \mathrm{~mm}, \mathrm{D}_{2}=24 \mathrm{~mm}$ and $\mathrm{H}_{2}=53 \mathrm{~mm}$, as shown in Fig.2. The polished DTIRC is finally cut away from the $60 \mathrm{~mm}$ diameter fused silica rod. The $\mathrm{D}_{2}=24 \mathrm{~mm}$ planar output end of the DTIRC is also ground and polished. This end face is in direct contact with cooling water, which ensures an efficient light coupling to the rod. The water flow rate is 6 liter / min. The inner wall of the pumping cavity is bonded with a protected silver-coated aluminum foil with $94 \%$ reflectivity. The maximum contact between coolant and the rod is essential for the removal of the dissipated heat. Both fused silica material and cooling water are useful for partially preventing both UV solarization and IR heating to the laser rod.

\section{LASER OSCILLATION EXPERIMENTS}

For the solar irradiance of $890 \mathrm{~W} / \mathrm{m}^{2}$ in Lisbon area in August 2012, the $0.9 \mathrm{~m}$ diameter Fresnel lens collects $445 \mathrm{~W}$ solar power to its focal zone. The $4 \mathrm{~mm}$ diameter, $25 \mathrm{~mm}$ length, 1.0 at \% Nd:YAG single-crystal rod is supplied by Altechna Co.Ltd. The $4 \mathrm{~mm}$ diameter, $25 \mathrm{~mm}$ length, 0.1 at $\% \mathrm{Cr}: 1.0$ at $\% \mathrm{Nd}: Y A G$ ceramic rod is supplied by Konoshima Chemical Co. Ltd. Japan. The upper ends of both rods are directly deposited with HR coating which effectively reflects the laser emission wavelength ( $\mathrm{R}>99.8 \%$ @ $1064 \mathrm{~nm}$ ), while allows the passage of other pump wavelength ( $\mathrm{T}>95 \%$ @ $808 \mathrm{~nm})$. The lower ends are AR coated for $(\mathrm{R}<0.2 \% @ 1064 \mathrm{~nm})$. The laser resonator is formed by both a $-0.5 \mathrm{~m}$ RoC output coupler and the HR reflector. The output coupler is placed $100 \mathrm{~mm}$ away from the rod, as indicated by Fig. 2. The reflectivity of the output coupler varies between $94 \%$ and $98 \%$. The concentrated solar power at the entrance face of the DTIRC and output laser powers are respectively measured with a Molectron PowerMax 500D and a Thorlabs PM1100D laser power meters.

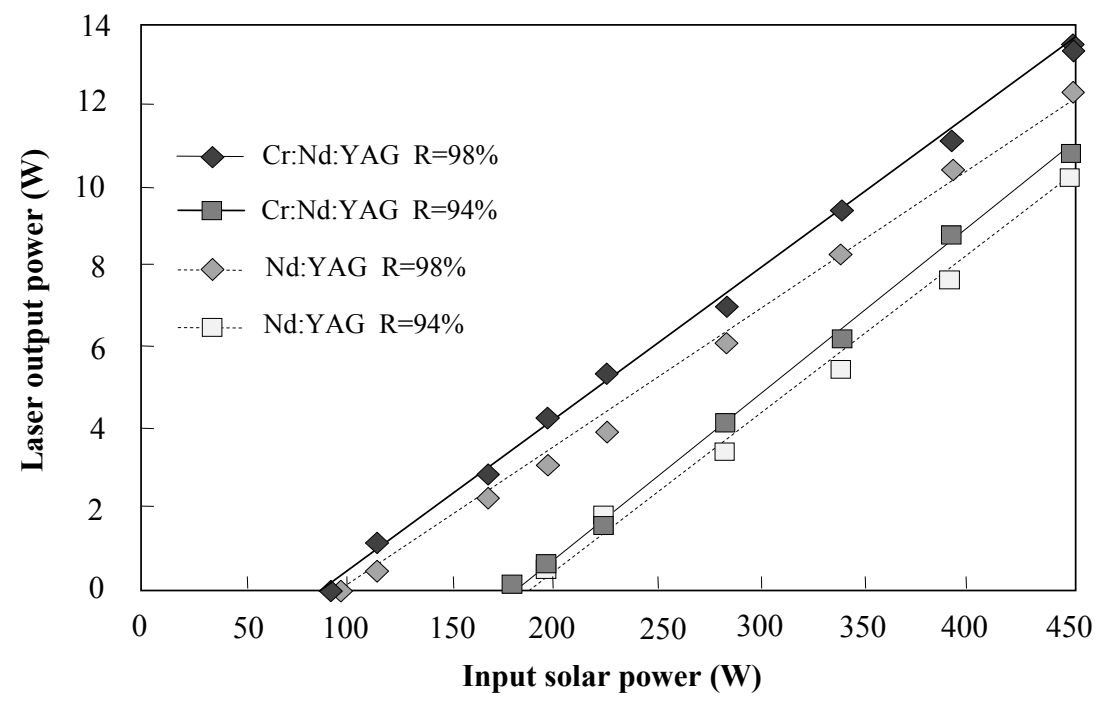

Fig.3. Solar laser output power versus concentrated solar power in the focal zone of the Fresnel lens. 
Return to the Manage Active Submissions page at http://spie.org/app/submissions/tasks.aspx and approve or disapprove this submission. Your manuscript will not be published without this approval. Please contact author_help@spie.org with any questions or concerns.

The $4 \mathrm{~mm}$ diameter Nd:YAG single-crystal rod is tested firstly. For $445 \mathrm{~W}$ solar power at the focus and a $-0.5 \mathrm{~m}$ RoC output coupler with $98 \%$ reflectivity, $12.3 \mathrm{~W}$ solar laser power is successfully measured [10], corresponding to $19.3 \mathrm{~W} / \mathrm{m}^{2}$ collection efficiency. By blocking progressively the incoming solar radiations with pizza slice type masks, placed in front of the Fresnel lens, different solar power levels are obtained and the laser output power can then be measured correspondingly. The threshold pump power of $94 \mathrm{~W}$ is measured in the focal zone. The slope efficiency of $3.5 \%$ is hence achieved. For $100 \mathrm{~mm}$ resonant cavity length and $-0.5 \mathrm{~m} \mathrm{RoC}$, a $24 \mathrm{~mm}$ diameter circularly symmetric laser beam profile is marked slowly on an opaque material, $1 \mathrm{~m}$ away from the output coupler. A $94 \%$ output coupler is also used to extract $10.1 \mathrm{~W}$ laser power. Despite the high threshold pump power of $187 \mathrm{~W}$, high slope efficiency of $3.9 \%$ is achieved. The threshold powers of both $94 \mathrm{~W}$ at $98 \%$ reflectivity and $187 \mathrm{~W}$ at $94 \%$ reflectivity for the $4 \mathrm{~mm}$ rod are used to calculate the round-trip loss of the laser resonant cavity. $1.7 \%$ round-trip loss is hence determined according to Findlay-Clay method [18]. Considering the absorption loss coefficient of $0.003 \mathrm{~cm}^{-1} @ 1064 \mathrm{~nm}$ for the Nd:YAG rod and $0.2 \%$ diffraction loss in LASCAD analysis, the $1.7 \%$ round-trip loss by Findlay-Clay analysis matches well with the sum of absorption and diffraction losses.

The $4 \mathrm{~mm}$ diameter $0.1 \mathrm{at} \% \mathrm{Cr}: 1.0$ at $\% \mathrm{Nd}: Y A G$ ceramic rod is finally tested. For $445 \mathrm{~W}$ solar power at the focus and a $-0.5 \mathrm{~m}$ RoC output coupler with $98 \%$ reflectivity, $13.5 \mathrm{~W}$ solar laser power is successfully measured, corresponding to $21.2 \mathrm{~W} / \mathrm{m}^{2}$ collection efficiency. The value is even $110 \%$ better than the maximum collection efficiency of $19.3 \mathrm{~W} / \mathrm{m}^{2}$ by the $4 \mathrm{~mm}$ diameter Nd:YAG single-crystal rod. Also by blocking progressively the incoming solar radiations with pizza slice type masks, placed in front of the Fresnel lens, different solar power levels are obtained and the laser output power can then be measured correspondingly. The threshold pump power of $89 \mathrm{~W}$ is measured in the focal zone. The slope efficiency of $3.78 \%$ is hence achieved. Another $-0.5 \mathrm{~m} \mathrm{RoC}, 94 \%$ output coupler is also used to extract $10.4 \mathrm{~W}$ laser power. Despite the high threshold pump power of $180 \mathrm{~W}$, high slope efficiency of $4.06 \%$ is achieved in this case.

A linear fiber-optic array for measuring the one-dimensional laser beam intensity distribution is placed, $5 \mathrm{~mm}$ and 1000 $\mathrm{mm}$ respectively, away from the output coupler along the optical axis of the laser rod. The $32 \mathrm{~mm}$ width, 128 optical fibers linear array is used to collect and transmit laser light to a Fairchild CCD 153A 512-element linear image sensor via a neutral density attenuator. This fiber-optic device has $0.25 \mathrm{~mm}$ core pitch resolution, so less than $2 \%$ laser beam diameter measurement error is found. This flexible fiber optic bundle has $2 \mathrm{~m}$ length. Outdoor solar laser beam diameter measurement is hence facilitated.

The laser beam divergence $\theta$ was found by adopting the Eq. (1):

$$
\arctan \theta=\frac{\phi_{2}-\phi_{1}}{2 L}
$$

Where $\phi_{1}$ and $\phi_{2}$ are the measured laser beam diameters at $1 / \mathrm{e}^{2}$ width, $5 \mathrm{~mm}$ and $1000 \mathrm{~mm}$ away from the output mirror respectively, and $L$ is the distance between these two points. $\mathrm{M}^{2}$ factor are then calculated as the ratio of the measured beam divergence $\theta$ and that of a diffraction-limited Gaussian beam of the same wavelength, which is $0.019^{\circ}$ for the Nd:YAG laser wavelength. Since both the Nd:YAG single-crystal and Cr:Nd:YAG ceramic rod have the same dimensions, $\mathrm{M}^{2}=30$ is separately determined for $-0.5 \mathrm{~m}$ RoC coupler in both cases. For $100 \mathrm{~mm}$ cavity length with a -2 $\mathrm{m} \mathrm{RoC}$, the laser beam divergence is largely reduced to $\mathrm{M}^{2}=12$. Despite the slight reduction of output laser power, significant increase in laser beam brightness is clearly noticeable by the fast marking rate on the opaque material.

\section{CONCLUSIONS}

Due to the superior light collection and concentration capability of both the DTIRC and the small conical pumping cavity, either the $4 \mathrm{~mm}$ diameter Nd:YAG single-crystal laser rod or the Cr:Nd:YAG ceramic laser rod is most efficiently pumped through the $0.9 \mathrm{~m}$ diameter Fresnel lens. $12.3 \mathrm{~W}, 13.5 \mathrm{~W}$ is produced respectively, corresponding to the collection efficiency of $19.3 \mathrm{~W} / \mathrm{m}^{2}$ for Nd:YAG rod, $21.2 \mathrm{~W} / \mathrm{m}^{2}$ for the Cr:Nd:YAG ceramic rod. The threshold pump power at the focus is as low as $94 \mathrm{~W}$ and $89 \mathrm{~W}$ respectively. The laser slope efficiency is also largely increased to $3.9 \%$ for the Nd:YAG rod, $4.06 \%$ for the Cr:Nd:YAG rod. The prototype solar laser is both small and powerful. Due to the low pump power threshold, the laser can be oscillated easily even on low irradiance days with thin clouds. On the one 
Return to the Manage Active Submissions page at http://spie.org/app/submissions/tasks.aspx and approve or disapprove this submission. Your manuscript will not be published without this approval. Please contact author_help@spie.org with any questions or concerns.

hand, the Nd:YAG single-crystal rods are produced by matured technology and exhibit much less solarization effect then the Cr:Nd:YAG ceramic rods, on the other hand, the Cr:Nd:YAG ceramic rod provides an enhanced laser performance whit adequate cooling.

\section{ACKNOWLEDGMENT}

This research project (PTDC/FIS/103599/2008) is funded by the Science and Technology Foundation of Portuguese Ministry of Science, Technology and Higher Education (FCT-MCTES).

\section{REFERENCES}

[1] Graham-Rowe, D., "Solar-powered laser,” Nat. Photonics 4, 64-65 (2010).

[2] Young, C. G., “A sun pumped cw one-watt laser,” Appl. Opt. 5(6), 993-997 (1966).

[3] Weksler, M. and Shwartz, J., "Solar-pumped solid-state lasers," IEEE J. Quantum Electron. 24(6), 1222$1228(1988)$.

[4] Arashi, H., Oka, Y., Sasahara, N., Kaimai, A. and Ishigame, M., “A solar-pumped cw 18 W Nd:YAG laser,” Jpn. J. Appl. Phys. 23 (8), 1051-1053 (1984).

[5] Benmair, R. M. J., Kagan, J., Kalisky, Y., Noter, Y., Oron, M., Shimony, Y. and Yogev, A., "Solar-pumped Er, Tm, Ho: YAG laser,” Opt. Lett. 15 (1), 36-38 (1990).

[6] Lando, M., Kagan, J., Linyekin, B. and Dobrusin, V., "A solar pumped Nd:YAG laser in the high collection efficiency regime," Opt. Commun. 222(1-6), 371-381 (2003).

[7] Cooke, D., "Sun-pumped lasers: revisiting an old problem with nonimaging optics," Appl. Opt. 31(36), 75417546 (1992).

[8] Yabe, T., Ohkubo, T., Uchida, S., Nakatsuka, M., Funatsu, T., Mabuti, A., Oyama, A., Nakagawa,Y., Oishi, T., Daito, K., Behgol, B., Nakayama,Y., Yoshida, M., Motokoshi, S., Sato, Y. and Baasandash, C., "High efficiency and economical solar energy pumped laser with Fresnel lens and chromium co-doped laser medium," Appl. Phys Lett. 90(26), 261120-3 (2007).

[9] Yabe, T., Bagheri, B., Ohkubo, T., Uchida, S., Yoshida, K., Funatsu, T., Oishi, T., Daito, K., Ishioka, M., Yasunaga, N., Sato, Y., Baasandash, C., Okamoto, Y., and Yanagitani, K., "100 W-class solar pumped laser for sustainable magnesium-hydrogen energy cycle," J. Appl. Phys. 104(8), 083104 -1-8 (2008).

[10] Liang, D. and Almeida, J., "Highly efficient solar pumped Nd:YAG laser,” Opt. Express 19(27), 26399-26405 (2011).

[11] Almeida, J., Liang, D. and Guillot, E., "Improvement in solar-pumped Nd:YAG laser beam brightness," Opt. Laser Technol. 44(7), 2115-2119 (2012).

[12] Dinh, T. H., Ohkubo, T., Yabe, T. and Kuboyama, H., "120 watt continuous wave solar-pumped laser with a liquid light-guide lens and a Nd:YAG rod,” Opt. Lett. 37(13), 2670-2672 (2012). 
Return to the Manage Active Submissions page at http://spie.org/app/submissions/tasks.aspx and approve or disapprove this submission. Your manuscript will not be published without this approval. Please contact author_help@spie.org with any questions or concerns.

[13] Liang, D., Almeida, J., and Guillot, E., "Side-pumped continuous-wave Cr:Nd:YAG ceramic solar laser," Appl. Phys. B - Lasers and Optics 111(2), 305-311( 2013).

[14] Liang, D. and Almeida, J., "Design of ultrahigh brightness solar-pumped disk laser," Appl. Opt. 51(26), 6382$6388(2012)$.

[15] Yagi, H., Yanagitani, T., Yoshida, H., Nakatsuka, M. and Ueda, K, "The optical properties and laser characteristics of $\mathrm{Cr}^{3+}$ and $\mathrm{Nd}^{3+}$ co-doped $\mathrm{Y}_{3} \mathrm{Al}_{5} \mathrm{O}_{12}$ ceramics," Opt. Laser Tech. 39(6), 1295-1300 (2007).

[16] Welford, W. T. and Winston, R., [High collection nonimaging optics], Academic Press, New York, (1989).

[17] Ning, X, Winston, R, and O'Gallagher, J., "Dielectric totally internally reflecting concentrators," Appl. Opt. 26(2), 300-305 (1987).

[18] Findlay, D. and Clay, R. A., "The measurement of internal losses in 4-level lasers," Phys. Lett. 20(3), 277-278 (1966). 